\title{
Etude de la variabilité des caractères de reproduction chez les petits ruminants en milieu d'élevage traditionnel au Sénégal
}

\author{
V. Clément ${ }^{1}$ J.P. Poivey ${ }^{1}$ O. Faugère ${ }^{2}$ E. Tillard ${ }^{3}$ \\ R. Lancelot ${ }^{4}$ A. Gueye ${ }^{4}$ D. Richard ${ }^{5}$ B. Bibé ${ }^{1}$
}

Mots-clés

Ovin - Caprin - Performance de reproduction - Paramètre génétique Intervalle entre parturitions - Age Facteur du milieu - Saison - Région Sénégal.

\begin{abstract}
Résumé
Une analyse génétique a été réalisée à partir des données recueillies lors du suivi individuel mis en place en milieu traditionnel au Sénégal dans le cadre du programme PPR (Pathologie et productivité des petits ruminants) par le CIRAD-EMVT (M ontpellier, France) et I'ISRA (Dakar, Sénégal). L'étude a été menée dans trois régions différentes, sur deux espèces : des ovins et des caprins. II s'agissait d'estimer les paramètres génétiques des caractères de reproduction des animaux : prolificité, intervalle entre mises bas et âge à la première mise bas. Le suivi individuel ayant été réalisé en milieu traditionnel, la variabilité environnementale était importante et les animaux étaient soumis à des conditions de milieu difficiles. La première étape a été d'identifier et de tester (à l'aide de modèles d'analyse de variance) les facteurs environnementaux de variation des performances de reproduction. Les facteurs fixes - village d'origine de l'animal, saison et année de mise bas (pour les caractères prolificité et intervalle entre mises bas) et saison et année de naissance (pour le caractère âge à la première mise bas) - ont été trouvés très significatifs. Pour le caractère prolificité, les facteurs fixes rang de mise bas et intervalle entre mises bas étaient significatifs, avec des tailles de portée qui augmentaient avec le rang de mise bas et qui étaient plus élevées pour un intervalle entre mises bas plus long. L'étape d'estimation de l'héritabilité et de la répétabilité a été réalisée selon un « modèle animal », en réalisant l'estimation des composantes de la variance par le maximum de vraisemblance restreinte. Chez les ovins, I'héritabilité était de $0,12 \pm 0,02$ pour la prolificité ; elle variait entre $0,07 \pm 0,02$ et $0,16 \pm 0,01$ pour l'intervalle entre mises bas et entre $0,07 \pm 0,07$ et $0,28 \pm 0,12$ pour l'âge à la première mise bas. Chez les caprins, I'héritabilité variait entre 0 et $0,08 \pm 0,02$ pour la prolificité, entre $0,02 \pm 0,03$ et $0,10 \pm 0,01$ pour l'intervalle entre mises bas et entre $0,12 \pm 0,06$ et $0,17 \pm 0,08$ pour l'âge à la première mise bas. Les résultats obtenus étaient moins élevés que ceux trouvés dans la bibliographie ; cela pourrait s'expliquer par le fait que les performances de reproduction, même corrigées pour les facteurs fixes significatifs, seraient influencées par des facteurs d'environnement non contrôlés. Il est aussi possible qu'il y ait antagonisme entre la production d'un grand nombre de descendants et la capacité à s'adapter aux conditions de milieu prévalantes au Sénégal.
\end{abstract}

1. INRA-SAGA, BP 27, 31326 Castanet-Tolosan Cedex, France

2. Mission française de coopération et d'action culturelle, BP 12090, Niamey, Niger

3. CIRAD-Elevage, 97410 St-Pierre, La Réunion

4. ISRA-LNERV, BP 2057, Dakar Hann, Sénégal

5. CIRAD-EMVT, Campus international de Baillarguet, BP 5035, 34032 Montpellier Cedex 1, France

\section{INTRODUCTION}

Un des moyens d'augmenter la productivité des troupeaux est d'avoir recours à l'amélioration génétique. Si ce moyen a fait ses preuves dans les pays tempérés, il reste peu répandu dans les pays tropicaux car la situation économique de ces pays (moyens financiers et humains) et les contraintes zoosanitaires n'ont guère per- 
mis d'envisager un programme de sélection d'une population en situation naturelle d'élevage, c'est-à-dire une sélection collective organisée entre éleveurs (par opposition à une sélection centralisée en station, beaucoup plus répandue).

Le programme PPR (Pathologie et productivité des petits ruminants) mis en place par l'IEMVT (Maisons-Alfort, France) et l'ISRA (Dakar, Sénégal) en 1983 a été élaboré pour approfondir les connaissances sur l'élevage ovin et caprin du Sénégal. L'objectif initial était d'établir un référentiel technico-économique des pratiques d'élevage de différentes zones de ce pays afin de définir des moyens efficaces et pertinents (sanitaires, zootechniques, etc.) pour améliorer la productivité du cheptel. A cet effet, un suivi individuel a été mis en place. Il repose sur un recueil régulier (à intervalle de quinze jours) d'informations démographiques, sanitaires et de croissance pondérale sur des animaux identifiés individuellement. Les données ont été vérifiées et rassemblées dans une base de données gérée localement sur micro-ordinateur. La méthode de suivi et les aspects informatiques ont été décrits par Faugère et coll. $(10,11)$.

C'est dans le cadre de ce suivi individuel du cheptel qu'une étude génétique a été entreprise. L'objectif de cette étude a été d'estimer l'héritabilité des caractères de reproduction de l'ensemble des ovins et des caprins suivis (les types génétiques ayant été identifiés et enregistrés), à partir de la base de données décrite ci-dessus. Ces estimations devaient permettre par ailleurs de vérifier la pertinence d'une action de sélection en milieu traditionnel. Les principales particularités des données étaient les suivantes :

- la variabilité environnementale était importante : elle s'est exprimée au niveau du climat, de la végétation, mais aussi des systèmes d'élevage ;

- les animaux étaient répartis en troupeaux (parfois de faible effectif) et chaque village comptait plusieurs troupeaux ;

- enfin, la connaissance des généalogies s'est limitée à la filiation maternelle, les pères étant inconnus.

\section{MATERIEL ET METHODES}

\section{L'animal et son milieu}

\section{Les caractéristiques géographiques}

Trois régions différentes ont été étudiées : la région de Louga, au Nord du Sénégal, correspondant à un climat sahélien, la région de Kaymor dans la partie centrale du pays, pour lequel le climat est de type sahélo-soudanien, et la région de Kolda, en Haute Casamance, sous un climat soudano-guinéen (figure 1) $(8,9)$. L'importance des précipitations et la durée de la saison des pluies diffèrent d'une zone à l'autre avec une augmentation de l'hygrométrie et de la pluviosité quand on passe du Nord au Sud du pays.

$\mathrm{Au}$ Nord du pays, la végétation est constituée par la savane (graminées annuelles), mais celle-ci disparaît presque totalement en saison sèche, laissant le sol le plus souvent à nu. La strate arbustive est représentée par des acacias. Dans la partie centrale, la végétation, de type savane arbustive, laisse de plus en plus souvent la place aux cultures. Dans le Sud du pays, le couvert végétal est de type savane boisée avec des graminées vivaces ; le paysage est découpé par des vallées au fond desquelles se trouvent des rizières.

\section{Les espèces et les races locales}

$\mathrm{Au}$ Nord, la population animale est constituée par des animaux de grand format, moutons Peuls-Peuls et chèvres Sahéliennes (8),

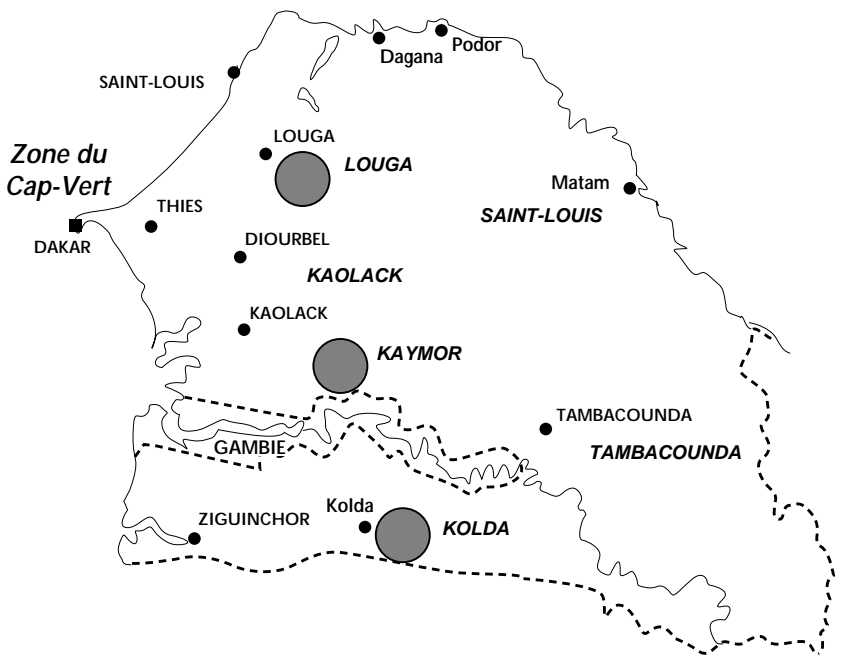

Zones d'implantation des suivis de petits ruminants (ovins et caprins) - Sénégal

Figure 1 : carte du Sénégal et localisation des zones de suivi des petits ruminants (ovins et caprins) par le programme PPR.

alors qu'au Sud, on rencontre des animaux plus petits, trypanotolérants : les moutons Djallonké et les chèvres Guinéennes (9). Dans la partie centrale, les ovins et les caprins résultent de croisements anciens entre les races du Nord et celles du Sud.

\section{Les pratiques d'élevage}

Les pratiques d'élevage présentent une variabilité entre régions, entre villages et entre éleveurs. L'unité d'élevage est le troupeau de concession. Une concession regroupe l'ensemble des habitations abritant les membres d'une même famille (18). Les éleveurs possèdent généralement les deux espèces, à l'exception des Wolofs (ethnie majoritaire dans le Nord du pays) qui élèvent uniquement des ovins. Pendant la journée, les troupeaux sont mixtes (ovins et caprins) ; la nuit, les deux espèces peuvent être séparées (c'est toujours le cas à Kolda, au Sud du pays), ou bien cohabiter. Les pratiques d'élevage ont été décrites par Faugère et coll. $(8,9)$, Moulin (18) et Moulin et coll. (19).

La finalité de l'élevage est multiple. Les petits ruminants sont élevés principalement pour subvenir aux besoins de la famille en lait et en viande. Chez les Peuls, les troupeaux ont souvent une orientation laitière : le lait est fourni par les caprins et consommé tel quel. Chez les Wolofs, ce sont les brebis qui fournissent le lait, l'élevage de caprins étant beaucoup moins répandu. En ce qui concerne la viande, elle est fournie par des moutons mâles qui ont été mis à l'embouche. Outre la production de lait et de viande, les petits ruminants (ovins et caprins) ont aussi une fonction socioculturelle et un rôle d'épargne importants : les éleveurs investissent en achetant des animaux et l'argent peut à nouveau être rapidement mobilisé en cas de besoin par la vente des animaux.

La reproduction est peu maîtrisée (aussi bien chez les caprins que chez les ovins), et des croisements entre apparentés peuvent se produire. La majorité des mâles sont exploités avant l'âge d'un an, et les reproducteurs sont de jeunes mâles issus du troupeau ou d'un troupeau voisin. Il arrive que l'éleveur fasse saillir les femelles du troupeau par un mâle reproducteur qui lui a été prêté ou loué. Ce reproducteur est alors choisi sur sa conformation (chez les ovins, les animaux de grande taille et à la robe blanche sont souvent préférés). 
Le taux de renouvellement des brebis et des chèvres est variable selon l'ethnie à laquelle appartient l'éleveur. Quelques femelles du troupeau peuvent être vendues et remplacées par l'achat de meilleures reproductrices (8). Cependant cette pratique reste peu répandue et il peut arriver qu'une femelle soit gardée jusqu'à l'âge de 8 ou 9 ans (9).

\section{L'unité d'étude}

Dans chaque zone étudiée, un certain nombre de villages ont été sélectionnés pour participer au suivi. Ces villages sont situés sur un périmètre de 20 à $40 \mathrm{~km}$ de diamètre et ils correspondent à des écosystèmes différents (zone de savane, zone de forêt, bordure d'un fleuve, etc.). Pour chacun des villages, certains troupeaux de concession ont été choisis (après accord avec les éleveurs) pour participer au recueil individuel des performances. Tous les animaux d'un troupeau ont été identifiés par des boucles auriculaires à usage unique et ont été suivis individuellement.

Dans cette étude, le choix de l'« unité statistique »d'analyse des données a été privilégié à un choix d' "unité zootechnique », pour un problème d'effectif : étant donné que plus de 50 p. 100 des troupeaux comprenaient moins de 20 animaux (figure 2) lors des analyses, les animaux ont été regroupés en unités plus grandes, les villages. Des analyses préliminaires ont montré que la prise en compte d'un effet troupeau intra-village diminuait la précision des estimations, du fait d'un dispositif totalement déséquilibré (cer-
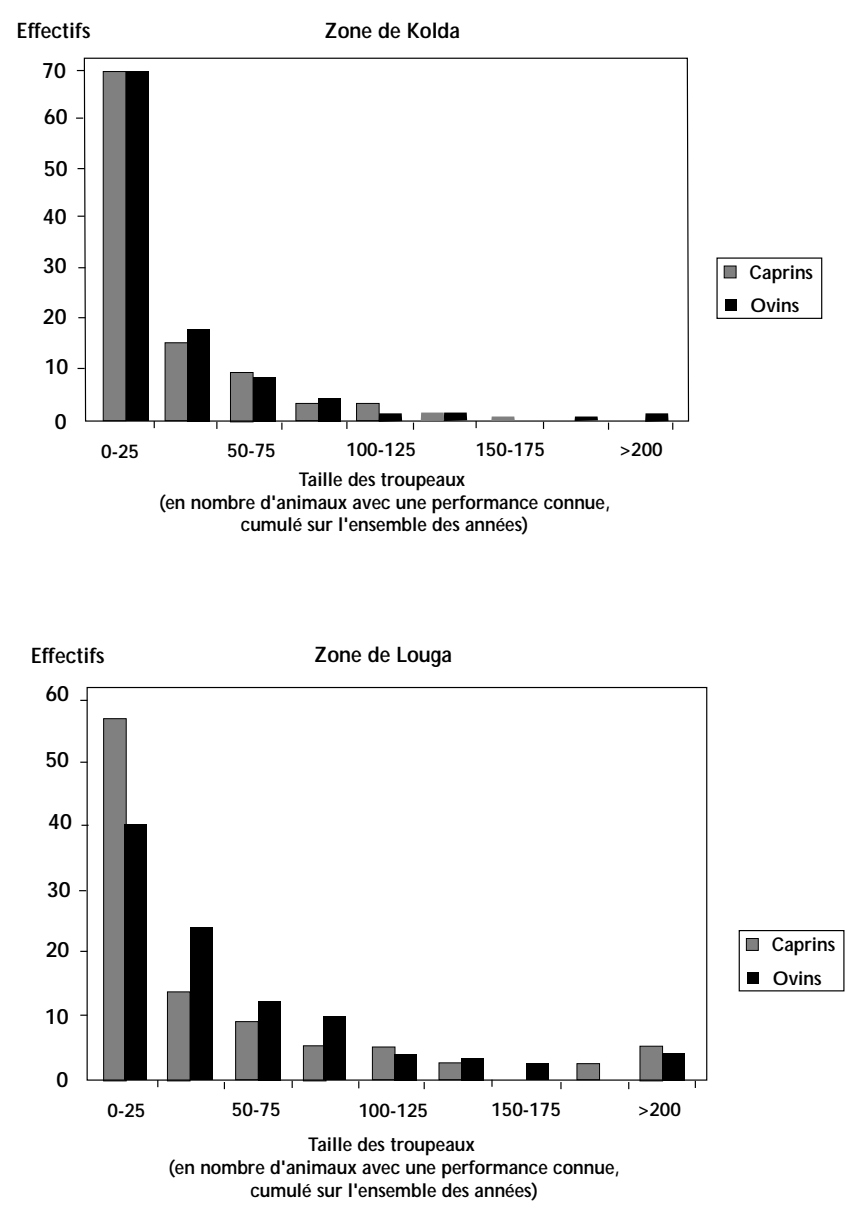

Figure 2 : distribution de la taille des troupeaux, en nombre d'animaux avec une performance connue, cumulé sur l'ensemble des années (de 1984 à 1995) dans les zones de suivi de Louga et de Kolda. tains niveaux des effets fixes contenaient seulement un ou deux éléments). Le regroupement des animaux d'un même village sousentend l'hypothèse que les conditions d'élevage intra-village sont semblables. Les pratiques d'élevage peuvent être différentes d'un éleveur à l'autre, notamment au niveau de la conduite sur pâturage, de la complémentation alimentaire et du logement. Cependant, les auteurs ont supposé que ces différences n'étaient pas à l'origine d'une variabilité importante des performances de reproduction et que la principale source de variation était le village. La prise en compte du facteur ethnie était envisageable pour la zone de Louga puisque dans cette région deux ethnies cohabitent (les Peuls et les Wolofs), et les pratiques d'élevage présentent une certaine homogénéité entre les éleveurs d'une même ethnie. Mais, des analyses de variance (tableaux I et II) ont montré que la variabilité prise en compte par un modèle avec un effet fixé ethnie est faible par rapport à un modèle comprenant un effet village (les coefficients de détermination sont en moyenne deux fois plus élevés dans cette deuxième situation). D'autre part, le modèle considérant un effet village intra-ethnie n'est pas meilleur que celui comprenant un effet village : les coefficients de détermination sont les mêmes pour ces deux types de modèles (tableaux I et II).

\section{Les performances de reproduction}

Les mises bas chez les ovins et chez les caprins peuvent avoir lieu toute l'année, mais elles sont plus fréquentes à certaines périodes de l'année $(8,9)$. Dans le Sud, la distribution était globalement la même chez les deux espèces (figure 3 ). Il y a deux maxima : un de mars à mai et l'autre de septembre à novembre, ce qui représente 65 p. 100 des mises bas. Dans le Nord, 75 p. 100 des naissances se répartissaient entre novembre et mars, le maximum se situant en janvier pour les ovins et en février pour les caprins (figure 3).

La prolificité était plus élevée et plus variable chez les races du Sud que chez celles du Nord (ovins et caprins) et elle était plus grande et plus variable chez les caprins que chez les ovins. L'intervalle entre mises bas était plus court et les animaux étaient plus précoces dans le Sud que dans le Nord (tableau III).
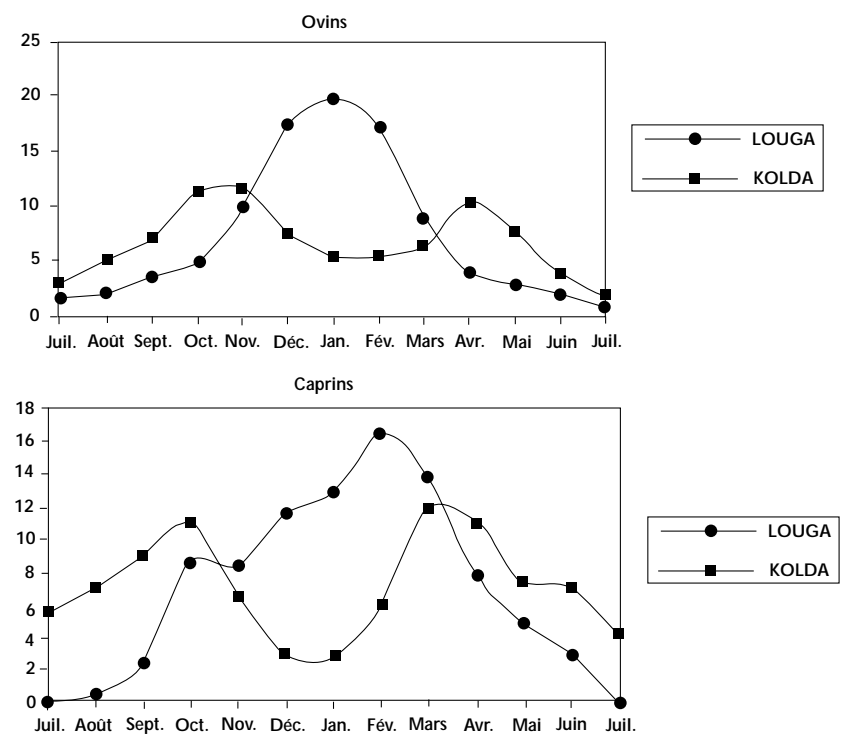

Figure 3 : répartition saisonnière des mises bas chez les ovins et les caprins des zones de suivi de Louga et de Kolda. 
Tableau I

Résultats des analyses de variance (modèles à effets fixés) pour différents modèles testés pour les ovins de la zone de suivi de Louga

\begin{tabular}{|c|c|c|c|c|c|c|}
\hline Caractère & Modèles & Pr. & $\mathbf{R}^{2}$ & Modèles & Pr. & $\mathbf{R}^{2}$ \\
\hline \multirow{11}{*}{$\begin{array}{l}\text { Intervalle } \\
\text { entre } \\
\text { mises bas }\end{array}$} & VIL & $* *$ & \multirow[t]{5}{*}{0,13} & VIL(ETN) & $* *$ & \multirow[t]{5}{*}{0,13} \\
\hline & SMB & ** & & SMB & ** & \\
\hline & YMB & $* *$ & & YMB & $* *$ & \\
\hline & SMB*YMB*VIL & ** & & SMB*YMB*VIL(ETN) & ** & \\
\hline & IN TER(RG MB) & $* *$ & & INTER(RGMB) & $* *$ & \\
\hline & ETN & ns & \multirow[t]{6}{*}{0,05} & VIL & $* *$ & \multirow[t]{6}{*}{0,13} \\
\hline & SM B & $* *$ & & ETN & ns & \\
\hline & YMB & ** & & SMB & $* *$ & \\
\hline & SMB*YMB*VIL & ** & & YMB & $* *$ & \\
\hline & INTER(RG MB) & $* *$ & & SMB*YMB*VIL & $* *$ & \\
\hline & & & & INTER(RGMB) & $* *$ & \\
\hline \multirow{9}{*}{$\begin{array}{l}\text { Age à la } \\
\text { première } \\
\text { mise bas }\end{array}$} & VIL & $* *$ & \multirow[t]{4}{*}{0,26} & VIL(ETN) & $* *$ & \multirow[t]{4}{*}{0,26} \\
\hline & SNAI & ns & & SNAI & ns & \\
\hline & YNAI & $* *$ & & YNAI & $* *$ & \\
\hline & SNAI*YNAI*VIL & $* *$ & & SNAI*YNAI*VIL(ETN ) & $* *$ & \\
\hline & ETN & ns & \multirow[t]{5}{*}{0,04} & VIL & $* *$ & \multirow[t]{5}{*}{0,26} \\
\hline & SNAI & ns & & ETN & ns & \\
\hline & YNAI & ns & & SNAI & ns & \\
\hline & \multirow{2}{*}{ SN AI*YNAI*ETN } & \multirow[t]{2}{*}{ ns } & & YNAI & $* *$ & \\
\hline & & & & SNAI*YNAI*VIL & $* *$ & \\
\hline
\end{tabular}

ns : non significatif ; $*$ : probabilité $<0,05 ; * *$ : probabilité $<0,01$

SMB : saison de mise bas ; YMB : année de mise bas ; SNAI : saison de naissance ; YNAI : année de naissance ; VIL : village ; ETN : ethnie ; RGMB : rang de mise bas INTER : première mise bas : âge à la mise bas ; mises bas suivantes : intervalle depuis la mise bas précédente

\section{Les analyses statistiques}

\section{Les caractères étudiés}

Trois caractères ont été choisis pour analyser les performances de reproduction :

- la prolificité ou taille de portée qui correspond au nombre de produits nés à terme de la gestation (vivants ou morts). Les tailles de portée nulles n'ont pas été prises en compte, car elles correspondaient à des avortements ;

- l'intervalle entre mises bas qui, dans ce cas, correspond à l'intervalle de temps (exprimé en mois) entre la mise bas considérée et la mise bas précédente ;

- l'âge de la femelle à la première mise bas (exprimé en mois), désigné aussi dans le texte par précocité.

Pour les deux dernières variables (prolificité et intervalle entre mises bas), il a été tenu compte des avortements lorsqu'ils ont pu être identifiés. Dans la base de données, les avortements ont été saisis au même titre que les mises bas (avec une taille de portée nulle). Aussi, les tailles de portée nulles, ainsi que les intervalles entre mises bas qui ont suivi et précédé un avortement, n'ont pas été pris en compte dans les analyses statistiques. Cependant, dans ces systèmes d'élevage, le nombre d'avortements était sous-estimé, ces derniers passant souvent inaperçus. Ainsi, lorsque la mise bas considérée faisait suite à un avortement non détecté, les intervalles entre mises bas se trouvaient rallongés, couvrant deux périodes de gestation dont une incomplète. Pour la même raison, l'âge à la première mise bas pouvait être rallongé s'il incluait une période de gestation conduisant à un avortement non détecté.
Pour l'intervalle entre mises bas et l'âge à la première mise bas, la méthode des rangs a été utilisée pour normaliser la distribution (3). Les méthodes classiques de normalisation (logarithme, racine carrée) n'étaient pas adaptées à la distribution bimodale observée pour ces deux caractères. Le principe de la méthode des rangs est le suivant : les valeurs sont classées et un rang leur est attribué, puis ce rang subit la transformation suivante (proposée par Blom, 3) :

$$
y_{i}=\Phi^{-1}\left(r_{i}-3 / 8\right) /(n+1 / 4)
$$

où $\Phi^{-1}$ est la fonction probit,

$\mathrm{r}_{\mathrm{i}}$ est le rang de la i ième observation,

$n$ est le nombre d'observations non manquantes.

L'analyse a été faite intra-zone (Louga, Kaymor, Kolda) et intraespèce (ovins, caprins). Le caractère prolificité a été étudié uniquement dans les cas où la variabilité était suffisante. Ainsi, les données relatives aux ovins de Louga et aux ovins de Kaymor pour lesquels la taille de portée était très proche de 1 et l'écarttype inférieur à 0,35 (ovins de Louga : 1,04 $\pm 0,19$; ovins de Kaymor : $1,11 \pm 0,33$ ) n'ont pas été analysées pour la prolificité.

\section{La méthode d'analyse et d'estimation}

La première étape (pré-analyse) a été faite en vue de rechercher les facteurs (effets fixes) qui avaient le plus d'incidence sur les variables étudiées, afin de les conserver dans le modèle d'analyse (modèle mixte). C'est donc un modèle à effets fixes (procédure GLM du logiciel SAS (Statistical analysis system, 32)) qui a été utilisé pour cette pré-analyse. Ceci était indispensable, notamment pour réduire le nombre de niveaux d'interaction entre effets fixes à retenir. Seuls les facteurs fixes significatifs (avec une probabilité de 5 p. 100) ont été retenus pour l'estimation des composantes de la variance. 


\section{Tableau II}

Résultats des analyses de variance (modèles à effets fixés) pour différents modèles testés pour les caprins de la zone de suivi de Louga

\begin{tabular}{|c|c|c|c|c|c|c|}
\hline Caractère & Modèles & Pr. & $\mathbf{R}^{2}$ & Modèles & Pr. & $\mathbf{R}^{2}$ \\
\hline \multirow[t]{11}{*}{ Prolificité } & VIL & $* *$ & \multirow[t]{5}{*}{0,19} & VIL(ETN) & $* *$ & \multirow[t]{5}{*}{0,19} \\
\hline & SM B & $*$ & & SMB & $*$ & \\
\hline & YMB & ** & & YMB & ** & \\
\hline & SMB*YMB*VIL & $* *$ & & SM B*YMB*VIL(ETN ) & $* *$ & \\
\hline & INTER(RGMB) & $* *$ & & INTER(RGMB) & $* *$ & \\
\hline & ETN & ns & \multirow[t]{6}{*}{0,14} & VIL & $* *$ & \multirow[t]{6}{*}{0,19} \\
\hline & SMB & ns & & ETN & ns & \\
\hline & YMB & $* *$ & & SMB & $*$ & \\
\hline & SMB*YMB*VIL & $* *$ & & YMB & $* *$ & \\
\hline & INTER(RGMB) & ** & & SMB*YMB*VIL & ** & \\
\hline & & & & INTER(RGMB) & $* *$ & \\
\hline \multirow{11}{*}{$\begin{array}{l}\text { Intervalle } \\
\text { entre } \\
\text { mises bas }\end{array}$} & VIL & $* *$ & \multirow[t]{5}{*}{0,14} & VIL(ETN) & $* *$ & \multirow[t]{5}{*}{0,14} \\
\hline & SMB & $* *$ & & SMB & $* *$ & \\
\hline & YMB & $* *$ & & YMB & $* *$ & \\
\hline & SMB*YMB*VIL & $* *$ & & SMB*YMB*VIL(ETN) & $* *$ & \\
\hline & INTER(RGMB) & $* *$ & & INTER(RGMB) & $* *$ & \\
\hline & ETN & ns & \multirow[t]{6}{*}{0,07} & VIL & $* *$ & \multirow[t]{6}{*}{0,14} \\
\hline & SM B & ns & & ETN & ns & \\
\hline & YMB & $* *$ & & SMB & $* *$ & \\
\hline & SM B*YMB*VIL & ** & & YMB & ** & \\
\hline & INTER(RGMB) & $* *$ & & SMB*YMB*VIL & $* *$ & \\
\hline & & & & INTER(RGMB) & $* *$ & \\
\hline \multirow{9}{*}{$\begin{array}{l}\text { Age à la } \\
\text { première } \\
\text { mise bas }\end{array}$} & VIL & $*$ & \multirow[t]{4}{*}{0,28} & VIL(ETN) & $*$ & \multirow[t]{4}{*}{0,28} \\
\hline & SNAI & ns & & SNAI & ns & \\
\hline & YNAI & $*$ & & YNAI & $*$ & \\
\hline & SNAI*YNAI*VIL & $* *$ & & SNAI*YNAI*VIL(ETN ) & $* *$ & \\
\hline & ETN & ns & \multirow[t]{5}{*}{0,10} & VIL & $*$ & \multirow[t]{5}{*}{0,28} \\
\hline & SNAI & ns & & ETN & ns & \\
\hline & YNAI & $* *$ & & SNAI & ns & \\
\hline & SN AI*YNAI*ETN & $* *$ & & YNAI & $*$ & \\
\hline & & & & SNAI*YNAI*VIL & $* *$ & \\
\hline
\end{tabular}

ns : non significatif ; $*$ : probabilité $<0,05 ; * *$ : probabilité $<0,01$

SMB : saison de mise bas ; YMB : année de mise bas ; VIL : village ; SNAI : saison de naissance ; YNAI : année de naissance ; ETN : ethnie ; RGMB : rang de mise bas ; INTER : première mise bas : âge à la mise bas ; mises bas suivantes : intervalle depuis la mise bas précédente

Les facteurs fixes significatifs (qui ont ensuite été pris en compte dans les modèles mixtes) sont les suivants :

- Pour le caractère prolificité

- le village, $\mathrm{t}_{\mathrm{j}}$;

- la saison de mise bas, $\mathrm{s}_{\mathrm{k}}$;

- l'année de mise bas, $\mathrm{y}_{1}$;

- l'interaction entre ces trois précédents facteurs, tsy $\mathrm{jkl}_{\mathrm{jl}}$;

- un effet appelé « inter », hiérarchisé dans le rang de mise bas, $\mathrm{r}_{\mathrm{m}}+\mathrm{i}_{\mathrm{mn}}$. Cet effet permet de tenir compte à la fois de la précocité de la femelle (âge à la première mise bas), de l'intervalle de temps qui s'est écoulé depuis la mise bas précédente et de la parité. Pour la première mise bas, l'effet fixe « inter » correspond à l'âge à la première mise bas. Pour les rangs de mise bas suivants, il correspond à l'intervalle entre mises bas.
- Pour le caractère intervalle entre mises bas

- le village, $\mathrm{t}_{\mathrm{j}}$;

- la saison de mise bas, $\mathrm{s}_{\mathrm{k}}$;

- l'année de mise bas, $\mathrm{y}_{1}$;

- l'interaction entre ces trois précédents facteurs, $\mathrm{tsy}_{\mathrm{jkl}}$;

- le rang de mise bas, $\mathrm{r}_{\mathrm{m}}$.

- Pour le caractère âge à la première mise bas

- le village, $\mathrm{t}_{\mathrm{j}}$;

- la saison de naissance, $\mathrm{s}_{\mathrm{k}}$;

- l'année de naissance, $\mathrm{y}_{1}$;

- l'interaction entre ces trois précédents facteurs, tsy ${ }_{\mathrm{jkl}}$. 


\section{Tableau III}

Effectifs, valeurs moyennes et écart-type de la prolificité (en nombre de produits par mise bas), de l'intervalle entre mises bas (en mois) et de l'âge à la première mise bas (en mois) chez les ovins et chez les caprins des zones de suivi de Louga, de Kaymor et de Kolda

\begin{tabular}{|c|c|c|c|c|c|}
\hline & & & Louga & Kaymor & Kolda \\
\hline \multirow[t]{3}{*}{0 vins } & Prolificité & $\begin{array}{l}\text { Effectif } \\
\text { Moyenne } \pm \text { e.t.* } \\
\% \text { simples } \\
\% \text { doubles } \\
\% \text { triples }\end{array}$ & $\begin{array}{c}4352 \\
1,04 \pm 0,19 \\
96,2 \\
3,8 \\
0\end{array}$ & $\begin{array}{c}3558 \\
1,11 \pm 0,33 \\
88,7 \\
11,1 \\
0,2\end{array}$ & $\begin{array}{c}5366 \\
1,17 \pm 0,39 \\
82,9 \\
16,7 \\
0,4\end{array}$ \\
\hline & $\begin{array}{l}\text { Intervalle } \\
\text { entre } \\
\text { mises bas }\end{array}$ & $\begin{array}{l}\text { Effectif } \\
\text { Moyenne } \pm \text { e.t. } \\
\text { Minimum } \\
\text { Maximum }\end{array}$ & $\begin{array}{c}8553 \\
11,53 \pm 3,29 \\
5,03 \\
27,43\end{array}$ & $\begin{array}{c}2666 \\
8,83 \pm 2,44 \\
5,07 \\
19,47\end{array}$ & $\begin{array}{c}3875 \\
8,13 \pm 2,06 \\
5,03 \\
17,43\end{array}$ \\
\hline & $\begin{array}{l}\text { Age à la } \\
\text { première } \\
\text { mise bas }\end{array}$ & $\begin{array}{l}\text { Effectif } \\
\text { Moyenne } \pm \text { e.t. } \\
\text { Minimum } \\
\text { Maximum }\end{array}$ & $\begin{array}{c}1362 \\
17,81 \pm 4,37 \\
7,63 \\
28,45\end{array}$ & $\begin{array}{c}667 \\
15,60 \pm 3,48 \\
7,34 \\
24,47\end{array}$ & $\begin{array}{c}1318 \\
15,21 \pm 3,26 \\
6,97 \\
24,31\end{array}$ \\
\hline \multirow[t]{3}{*}{ Caprins } & Prolificité & $\begin{array}{l}\text { Effectif } \\
\text { M oyenne } \pm \text { e.t. } \\
\% \text { simples } \\
\% \text { doubles } \\
\% \text { triples } \\
\% \text { quadruples }\end{array}$ & $\begin{array}{c}4112 \\
1,17 \pm 0,38 \\
83,0 \\
16,8 \\
0,2 \\
0\end{array}$ & $\begin{array}{c}4706 \\
1,66 \pm 0,64 \\
42,6 \\
49,1 \\
8,0 \\
0,4\end{array}$ & $\begin{array}{c}4925 \\
1,56 \pm 0,64 \\
51,5 \\
41,3 \\
6,8 \\
0,4\end{array}$ \\
\hline & $\begin{array}{l}\text { Intervalle } \\
\text { entre } \\
\text { mises bas }\end{array}$ & $\begin{array}{l}\text { Effectif } \\
\text { Moyenne } \pm \text { e.t. } \\
\text { Minimum } \\
\text { Maximum }\end{array}$ & $\begin{array}{c}3133 \\
12,02 \pm 3,71 \\
5,03 \\
26,41\end{array}$ & $\begin{array}{c}3413 \\
9,09 \pm 2,37 \\
5,07 \\
18,42\end{array}$ & $\begin{array}{c}3149 \\
7,71 \pm 1,85 \\
5,03 \\
15,43\end{array}$ \\
\hline & $\begin{array}{l}\text { Age à la } \\
\text { première } \\
\text { mise bas }\end{array}$ & $\begin{array}{l}\text { Effectif } \\
\text { Moyenne } \pm \text { e.t. } \\
\text { Minimum } \\
\text { Maximum }\end{array}$ & $\begin{array}{c}954 \\
16,09 \pm 4,39 \\
7,96 \\
26,38\end{array}$ & $\begin{array}{c}1127 \\
12,39 \pm 3,08 \\
5,82 \\
22,27\end{array}$ & $\begin{array}{c}1468 \\
12,24 \pm 3,10 \\
5,59 \\
22,40\end{array}$ \\
\hline
\end{tabular}

* e.t. : écart-type

Pour la prolificité et l'intervalle entre mises bas, l'interaction village d'appartenance de la femelle*saison de mise bas*année de mise bas a été testée (facteur tsy $\mathrm{j}_{\mathrm{jk}}$ du modèle). La saison et l'année de mise bas ont été remplacées par la saison et l'année de naissance de la femelle pour le caractère âge à la première mise bas. La prise en compte d'un facteur combiné village*saison*année en plus de l'effet moyen des trois facteurs résulte de deux observations : d'une part, le rythme des saisons est variable d'une année sur l'autre et, d'autre part, les variations du facteur saison*année (alimentation, mode de conduite, prophylaxie, etc.) ne sont pas obligatoirement synchronisées entre les villages ; cela laisse penser à l'existence d'une interaction entre ces facteurs.

Pour le facteur village, le nombre de modalités varie de 9 à 28 selon la zone étudiée. Les facteurs saison de mise bas et saison de naissance comprennent deux modalités : une correspondant à la saison sèche, l'autre à la saison des pluies. Pour le facteur année, il y a 10 à 12 niveaux : de l'année 1983 à l'année 1995 en supprimant les années avec des effectifs trop faibles (inférieurs à 10). Pour l'interaction, il y a donc au maximum 28 x 2 x 12 niveaux.

Pour chacun des facteurs fixes, les niveaux qui présentaient des effectifs trop faibles ont été éliminés (facteurs village, saison, année) ou regroupés entre eux (facteurs « inter » et rang de mise bas) en prenant en compte l'homogénéité intra-classe.
L'estimation des paramètres génétiques et de la répétabilité a été faite selon un « modèle animal », en utilisant la méthode d'estimation des composantes de la variance par le maximum de vraisemblance restreinte (Restricted maximum likelihood ou REML, 22), à l'aide du logiciel VCE (12). Il s'agit de modèles mixtes d'analyse. Ces modèles sont les suivants :

- Pour le caractère prolificité

$$
\mathrm{Y}_{\mathrm{ijklmno}}=\mu+\mathrm{A}_{\mathrm{i}}+\mathrm{P}_{\mathrm{i}}+\mathrm{t}_{\mathrm{j}}+\mathrm{s}_{\mathrm{k}}+\mathrm{y}_{1}+\mathrm{tsy}_{\mathrm{jkl}}+\mathrm{r}_{\mathrm{m}}+\mathrm{i}_{\mathrm{mn}}+\mathrm{E}_{\mathrm{ijklmno}}
$$

où $\mathrm{Y}_{\mathrm{ijklmno}}$ est la o $\mathrm{o}^{\text {ième }}$ performance de l'individu $\mathrm{i}$, du village $\mathrm{j}$, de la saison k, de l'année 1, de la modalité n du facteur « inter » hiérarchisé dans le rang de mise bas $\mathrm{m}$;

$\mu$ est la moyenne générale de la population ;

$\mathrm{A}_{\mathrm{i}}$ est la valeur génétique additive de l'individu i ;

$\mathrm{P}_{\mathrm{i}}$ est l'effet environnement permanent de l'individu i ; c'est un effet aléatoire qui permet de prendre en compte la ressemblance entre les performances successives d'un même individu et à partir duquel sera estimée la répétabilité ;

$\mathrm{t}_{\mathrm{j}}$ est l'effet fixe village ;

$\mathrm{s}_{\mathrm{k}}$ est l'effet fixe saison de mise bas ; 
$\mathrm{y}_{1}$ est l'effet fixe année de mise bas ;

tsy $_{\mathrm{jkl}}$ est l'interaction village $(\mathrm{j}) *$ saison $(\mathrm{k}) *$ année $(\mathrm{l})$;

$r_{m}+i_{m n}$ est l'effet fixe inter (n) hiérarchisé dans le rang de mise bas $(\mathrm{m})$;

$\mathrm{E}_{\mathrm{ijklmno}}$ est l'effet résiduel.

- Pour le caractère intervalle entre mises bas

$$
\mathrm{Y}_{\mathrm{ijklmno}}=\mu+\mathrm{A}_{\mathrm{i}}+\mathrm{P}_{\mathrm{i}}+\mathrm{t}_{\mathrm{j}}+\mathrm{s}_{\mathrm{k}}+\mathrm{y}_{\mathrm{l}}+\text { tsy }_{\mathrm{jkl}}+\mathrm{r}_{\mathrm{m}}+\mathrm{E}_{\mathrm{ijklmno}}
$$

où $\mathrm{Y}_{\mathrm{ijklmno}}$ est la o $\mathrm{o}^{\text {ième }}$ performance de l'individu $\mathrm{i}$, du village $\mathrm{j}$, de la saison $\mathrm{k}$, de l'année 1 , de la modalité $\mathrm{n}$ du facteur « inter » hiérarchisé dans le rang de mise bas $\mathrm{m}$;

$\mu$ est la moyenne générale de la population;

$\mathrm{A}_{\mathrm{i}}$ est la valeur génétique additive de l'individu i ;

$\mathrm{P}_{\mathrm{i}}$ est l'effet environnement permanent de l'individu i (effet aléatoire) ;

$\mathrm{t}_{\mathrm{j}}$ est l'effet fixe village ;

$\mathrm{s}_{\mathrm{k}}$ est l'effet fixe saison de mise bas ;

$\mathrm{y}_{1}$ est l'effet fixe année de mise bas ;

tsy $_{\mathrm{jkl}}$ est l'interaction village (j) * saison (k) * année (l);

$r_{m}$ est l'effet fixe rang de mise bas ;

$\mathrm{E}_{\mathrm{ijklmno}}$ est l'effet résiduel.

- Pour le caractère âge à la première mise bas

$$
\mathrm{Y}_{\mathrm{ijklmno}}=\mu+\mathrm{A}_{\mathrm{i}}+\mathrm{P}_{\mathrm{i}}+\mathrm{t}_{\mathrm{j}}+\mathrm{s}_{\mathrm{k}}+\mathrm{y}_{1}+\text { tsy }_{\mathrm{jkl}}+\mathrm{E}_{\mathrm{ijklmno}}
$$

où $\mathrm{Y}_{\mathrm{ijklmno}}$ est la o $\mathrm{o}^{\text {ième }}$ performance de l'individu $\mathrm{i}$, du village $\mathrm{j}$, de la saison k, de l'année 1, de la modalité $\mathrm{n}$ du facteur « inter » hiérarchisé dans le rang de mise bas $\mathrm{m}$; $\mu$ est la moyenne générale de la population ;

$\mathrm{A}_{\mathrm{i}}$ est la valeur génétique additive de l'individu i ;

$\mathrm{P}_{\mathrm{i}}$ est l'effet environnement permanent de l'individu i (effet aléatoire) ;

$\mathrm{t}_{\mathrm{j}}$ est l'effet fixe village ;

$\mathrm{s}_{\mathrm{k}}$ est l'effet fixe saison de naissance ;

$\mathrm{y}_{1}$ est l'effet fixe année de naissance ;

tsy $_{\mathrm{jkl}}$ est l'interaction village $(\mathrm{j}) *$ saison $(\mathrm{k}) *$ année $(\mathrm{l})$;

$\mathrm{E}_{\mathrm{ijklmno}}$ est l'effet résiduel.

L'intérêt de cette méthode de résolution est qu'elle prend en compte simultanément et sans biais les effets fixes et les effets aléatoires. Les parentés entre individus utilisées pour la résolution se limitent aux filiations maternelles.

\section{RESU LTATS}

La prolificité n'a pas été étudiée pour les ovins de Louga et de Kaymor, en raison d'une trop faible variabilité du caractère (tableau III).

\section{Facteurs de variation des performances de reproduction}

Tous les facteurs pris en compte dans les analyses de variance (modèles à effets fixes) sont très significatifs (tableaux IV, V, VI). Les coefficients de détermination varient entre 0,21 et 0,36 pour la prolificité, entre 0,13 et 0,22 pour l'intervalle entre mises bas, et entre 0,28 et 0,45 pour l'âge à la première mise bas.

\section{Tableau IV}

\begin{tabular}{|c|c|c|c|c|c|c|}
\hline Région & Espèce & Nb. obs. & Moy. & $\mathbf{R}^{2}$ & Effets fixes & Pr. \\
\hline Louga & Caprins & 4352 & 1,17 & 0,19 & $\begin{array}{l}\text { SMB } \\
\text { YMB } \\
\text { VIL } \\
\text { SMB*YMB*VIL } \\
\text { INTER(RGMB) }\end{array}$ & $\begin{array}{l}* \\
* * \\
* * \\
* * \\
* *\end{array}$ \\
\hline Kaymor & Caprins & 4706 & 1,66 & 0,36 & $\begin{array}{l}\text { SMB } \\
\text { YMB } \\
\text { VIL } \\
\text { SMB*YMB*VIL } \\
\text { INTER(RGMB) }\end{array}$ & $\begin{array}{l}\text { ns } \\
* \\
* * \\
* \\
* *\end{array}$ \\
\hline Kolda & Ovins & 5366 & 1,18 & 0,21 & $\begin{array}{l}\text { SMB } \\
\text { YMB } \\
\text { VIL } \\
\text { SMB*YMB*VIL } \\
\text { INTER(RGMB) }\end{array}$ & $\begin{array}{l}\text { ns } \\
\text { ns } \\
* * \\
\text { ns } \\
* *\end{array}$ \\
\hline Kolda & Caprins & 4925 & 1,57 & 0,36 & $\begin{array}{l}\text { SMB } \\
\text { YMB } \\
\text { VIL } \\
\text { SMB*YMB*VIL } \\
\text { INTER(RGMB) }\end{array}$ & $\begin{array}{l}\text { ns } \\
* * \\
* * \\
\text { ns } \\
* *\end{array}$ \\
\hline
\end{tabular}

Résultats des analyses de variance pour la prolificité (modèles à effets fixes)

ns : non significatif ; $*$ : probabilité $<0,05 ;{ }^{* *}$ : probabilité $<0,01$

SMB : saison de mise bas ; YMB : année de mise bas ; VIL : village ; RGMB : rang de mise bas ; INTER : première mise bas : âge à la mise bas ; mises bas suivantes : intervalle depuis la mise bas précédente 
Tableau V

Résultats des analyses de variance pour l'intervalle entre mises bas (modèles à effets fixes)

\begin{tabular}{|c|c|c|c|c|c|c|}
\hline Région & Espèce & Nb. obs. & Moy. & $\mathbf{R}^{2}$ & Effets fixes & Pr. \\
\hline Louga & O vins & 8553 & 11,60 & 0,13 & $\begin{array}{l}\text { SMB } \\
\text { YMB } \\
\text { VIL } \\
\text { SMB*YMB*VIL } \\
\text { RGMB }\end{array}$ & $\begin{array}{l}* * \\
* * \\
* * \\
* * \\
* *\end{array}$ \\
\hline Louga & Caprins & 3133 & 12,14 & 0,14 & $\begin{array}{l}\text { SMB } \\
\text { YMB } \\
\text { VIL } \\
\text { SMB*YMB*VIL } \\
\text { RGMB }\end{array}$ & $\begin{array}{l}* * \\
* * \\
* * \\
* * \\
* *\end{array}$ \\
\hline Kaymor & O vins & 2666 & 8,94 & 0,22 & $\begin{array}{l}\text { SMB } \\
\text { YMB } \\
\text { VIL } \\
\text { SMB*YMB*VIL } \\
\text { RGMB }\end{array}$ & $\begin{array}{l}* * \\
* * \\
* * \\
* * \\
* *\end{array}$ \\
\hline Kaymor & Caprins & 3413 & 9,20 & 0,20 & $\begin{array}{l}\text { SMB } \\
\text { YMB } \\
\text { VIL } \\
\text { SMB*YMB*VIL } \\
\text { RGMB }\end{array}$ & $\begin{array}{l}\text { ns } \\
* * \\
* * \\
* * \\
* *\end{array}$ \\
\hline Kolda & 0 vins & 3875 & 8,24 & 0,21 & $\begin{array}{l}\text { SMB } \\
\text { YMB } \\
\text { VIL } \\
\text { SMB*YMB*VIL } \\
\text { RGMB }\end{array}$ & $\begin{array}{l}\mathrm{ns} \\
\mathrm{ns} \\
* * \\
* * \\
* *\end{array}$ \\
\hline Kolda & Caprins & 3149 & 7,83 & 0,21 & $\begin{array}{l}\text { SMB } \\
\text { YMB } \\
\text { VIL } \\
\text { SMB*YMB*VIL } \\
\text { RGMB }\end{array}$ & $\begin{array}{l}\text { ns } \\
\text { ns } \\
* * \\
\text { ns } \\
* *\end{array}$ \\
\hline
\end{tabular}

ns : non significatif ; $*$ : probabilité $<0,05 ; * *$ : probabilité $<0,01$

VIL : village ; SMB : saison de mise bas ; YMB : année de mise bas ; RGMB : rang de mise bas

L'effet fixe village présente des estimées très importantes pour chacun de ses niveaux (figures 4 et 5). La saison et l'année de mise bas (caractère prolificité et intervalle entre mises bas), la saison et l'année de naissance (caractère âge à la première mise bas) sont des facteurs significatifs pour les performances de reproduction. La taille de portée est plus élevée lorsque la mise bas a lieu en saison sèche. Pour l'intervalle entre mises bas, la saison la plus favorable dépend de la région. Les intervalles entre mises bas sont plus faibles en saison sèche pour les régions du Centre et du Sud (Kaymor et Kolda) et plus faibles en saison des pluies pour le Nord (Louga). A Kaymor, l'intervalle entre mises bas a été calculé chez les ovins. La moyenne générale est de $8,7 \pm 2,7$ mois lorsque l'agnelage a lieu en saison sèche, contre 9,4 $\pm 2,7$ lorsqu'il a lieu en saison des pluies.

Le facteur « inter » a une influence significative sur les caractères étudiés. Pour la prolificité, on observe que les femelles qui mettent bas pour la première fois plus tardivement ont des tailles de portée plus élevées (figure 6). Par exemple, chez les caprins de Kolda, le pourcentage de portées multiples est de 13 p. 100 lorsque la première mise bas est comprise entre 6 et 10 mois, 16 p. 100 lorsqu'elle est comprise entre 10 et 18 mois, et 22 p. 100 lorsqu'elle est com- prise entre 18 et 22 mois. D'autre part, la prolificité augmente au cours de la vie de la femelle (figure 6). Par exemple, la taille d'une portée moyenne chez les ovins de Kolda passe de 1,05 à la première mise bas, à 1,27 à la cinquième et à 1,33 à la huitième. Pour le caractère intervalle entre mises bas, la performance est d'autant meilleure que le rang de mise bas est élevé, c'est-à-dire que l'intervalle entre mises bas diminue au cours de la vie de la femelle (figure 7).

\section{Estimation de l'héritabilité et de la répétabilité}

Les caractères étudiés sont faiblement à moyennement héritables (tableaux VII, VIII et IX). Chez les ovins, l'héritabilité est de 0,12 pour la prolificité, elle varie entre 0,07 et 0,16 pour l'intervalle entre mises bas et 0,07 et 0,28 pour l'âge à la première mise bas. Chez les caprins, l'héritabilité varie entre 0 et 0,08 pour la prolificité, 0,02 et 0,10 pour l'intervalle entre mises bas et 0,12 et 0,17 pour l'âge à la première mise bas. Les caractères sont moyennement répétables. En effet, chez les ovins, la répétabilité est de 0,16 pour la prolificité et de 0,07 à 0,16 pour l'intervalle entre mises bas ; chez les caprins, de 0,10 à 0,13 pour la prolificité et de 0,06 à 0,10 pour l'intervalle entre mises bas. 
Tableau VI

Résultats des analyses de variance pour l'âge à la première mise bas (modèles à effets fixes)

\begin{tabular}{|c|c|c|c|c|c|c|}
\hline Région & Espèce & Nb. obs. & Moy. & $\mathbf{R}^{2}$ & Effets fixes & Pr. \\
\hline Louga & O vins & 1362 & 18,05 & 0,26 & $\begin{array}{l}\text { SNAI } \\
\text { YNAI } \\
\text { VIL } \\
\text { SNAI*YNAI*VIL }\end{array}$ & $\begin{array}{l}\text { ns } \\
* * \\
* * \\
* *\end{array}$ \\
\hline Louga & Caprins & 974 & 16,47 & 0,28 & $\begin{array}{l}\text { SNAI } \\
\text { YNAI } \\
\text { VIL } \\
\text { SNAI*YNAI*VIL }\end{array}$ & $\begin{array}{l}\text { ns } \\
* \\
* \\
* *\end{array}$ \\
\hline Kaymor & $O$ vins & 667 & 15,63 & 0,35 & $\begin{array}{l}\text { SNAI } \\
\text { YNAI } \\
\text { VIL } \\
\text { SNAI*YNAI*VIL }\end{array}$ & $\begin{array}{l}* \\
\text { ns } \\
* \\
* *\end{array}$ \\
\hline Kaymor & Caprins & 1127 & 12,59 & 0,35 & $\begin{array}{l}\text { SNAI } \\
\text { YNAI } \\
\text { VIL } \\
\text { SNAI*YNAI*VIL }\end{array}$ & $\begin{array}{l}* * \\
* * \\
* * \\
* *\end{array}$ \\
\hline Kolda & O vins & 1318 & 15,43 & 0,43 & $\begin{array}{l}\text { SNAI } \\
\text { YNAI } \\
\text { VIL } \\
\text { SNAI*YNAI*VIL }\end{array}$ & $\begin{array}{l}* * \\
* * \\
* * \\
* *\end{array}$ \\
\hline Kolda & Caprins & 1468 & 12,38 & 0,45 & $\begin{array}{l}\text { SNAI } \\
\text { YNAI } \\
\text { VIL } \\
\text { SNAI*YNAI*VIL }\end{array}$ & $\begin{array}{l}\text { ns } \\
* * \\
* * \\
* *\end{array}$ \\
\hline
\end{tabular}

ns : non significatif ; $*$ : probabilité $<0,05 ; * *$ : probabilité $<0,01$

VIL : village ; SNAI : saison de naissance ; YNAI : année de naissance

Pour le caractère intervalle entre mises bas, la variance d'environnement permanent est nulle (à l'exception des caprins de Kaymor), c'est-à-dire qu'il n'y a pas de ressemblance, autre que d'origine génétique, entre les performances successives d'un même individu. Dans ce cas, la répétabilité (somme de la variance génétique et de la variance d'environnement permanent, divisée par la variance totale) est alors égale à l'héritabilité.

\section{DISCUSSION}

\section{Facteurs de variation des performances de reproduction}

Dans cette étude, les facteurs de l'environnement ont une influence très nette sur les performances de reproduction, comme le montrent les seuils de signification des effets fixes.

\section{Effet de la saison sur les performances de reproduction}

Les performances pour la prolificité sont meilleures lorsque la mise bas a lieu en saison sèche. Les mises bas qui se produisent à cette époque correspondent à des fécondations de fin de saison d'hivernage, début de saison sèche, au moment où les ressources alimentaires sont en quantité importante et de bonne qualité. A Kolda, c'est aussi la période pendant laquelle les animaux qui ont été gardés à l'attache en saison des cultures sont relâchés. Par contre, les mises bas qui ont lieu pendant l'hivernage correspondent à des fécondations de fin de saison sèche début d'hivernage, période difficile car la quantité de fourrage disponible est réduite et les animaux sont en état de stress à l'arrivée des premières pluies. Ainsi l'alimentation pourrait être une des causes importantes de variation de la prolificité : une alimentation en quantité suffisante et de bonne qualité pourrait favoriser les naissances doubles (ou triples), avec pour conséquence une variation de la prolificité au cours de l'année (9).

Pour le caractère intervalle entre mises bas, les meilleures performances sont observées lorsque la mise bas a lieu en saison sèche pour les régions de Kaymor et de Kolda, et en saison des pluies pour Louga. Ces variations de l'intervalle entre mises bas (avec la mise bas précédente) peuvent être reliées à la répartition saisonnière des naissances. Il semblerait en effet que, malgré la présence continue des mâles dans les troupeaux, il existe des périodes plus favorables pour la réussite des saillies. A Kolda et à Kaymor où la répartition annuelle des naissances a une allure bimodale, les femelles qui ont eu des produits en fin de saison sèche (pendant le premier pic des naissances) peuvent mettre bas à nouveau au début de la saison sèche suivante (pendant le deuxième pic des naissances). L'intervalle sera alors inférieur à 8 mois. En revanche, si une femelle met bas pendant l'hivernage, la mise bas précédente n'a pas pu avoir lieu pendant le premier pic des naissances (de mars à mai), car l'intervalle serait alors inférieur à 6 mois, mais plutôt au moment du deuxième pic des naissances (octobre à décembre) de l'année précédente. L'intervalle mesuré pour cette dernière mise bas est alors de 9 ou 10 mois. A Louga, si une femelle a eu des produits en saison des pluies, la mise bas précédente peut avoir eu lieu à la saison sèche précédente. L'intervalle est alors plus court. Par contre, les femelles qui mettent bas en saison sèche attendront donc la saison sèche suivante (pendant laquelle la majorité des naissances ont lieu) pour mettre bas à nouveau. 


\section{Effectifs}

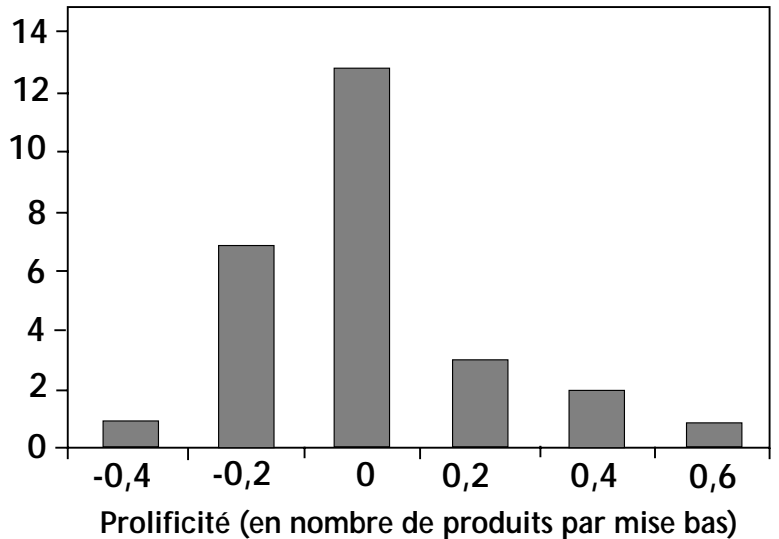

\section{Effectifs}

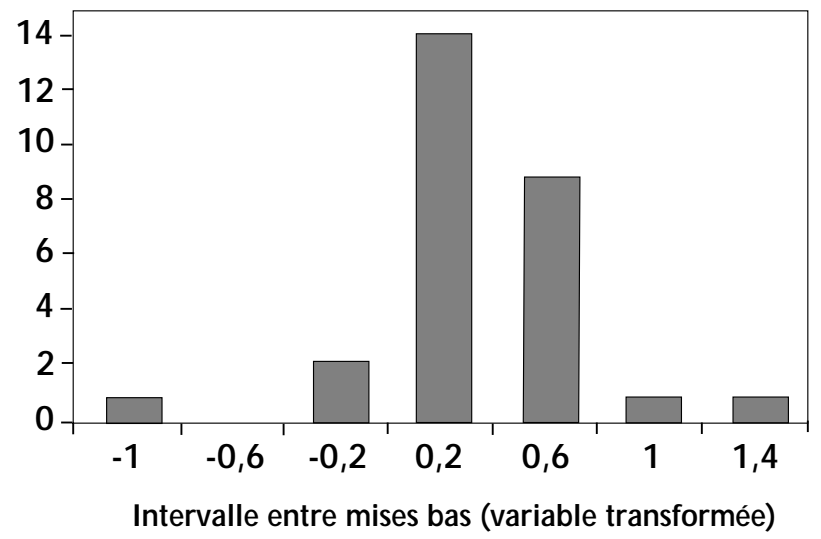

Effectifs

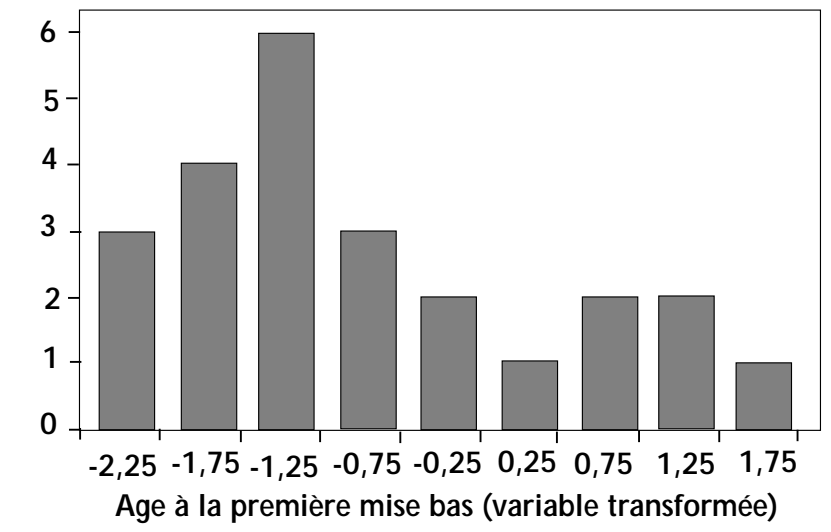

Figure 4 (ci-dessus) : histogramme des estimées de moindres carrés des modèles à effets fixes. Effet fixe village pour les ovins de la zone de suivi de Kolda. Variable transformée par la méthode des rangs : utilisation de la fonction probit (Blom G., 1958, NY, USA, John Wiley \& Sons).

Figure 5 (à droite) : histogramme des estimées de moindres carrés des modèles à effets fixes. Effet fixe village pour les caprins de la zone de suivi de Kolda. Variable transformée par la méthode des rangs : utilisation de la fonction probit (Blom $G$., 1958, NY, USA, John Wiley \& Sons).

\section{Effectifs}

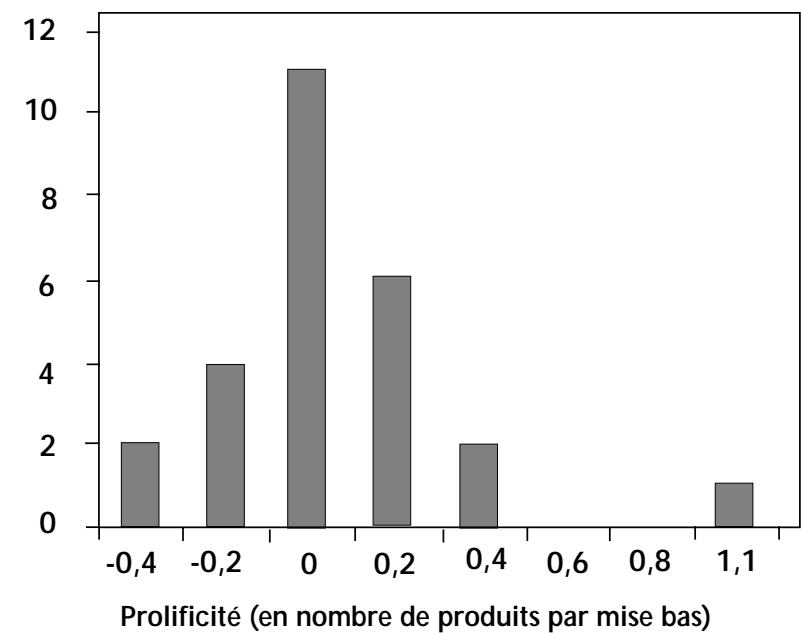

Effectifs

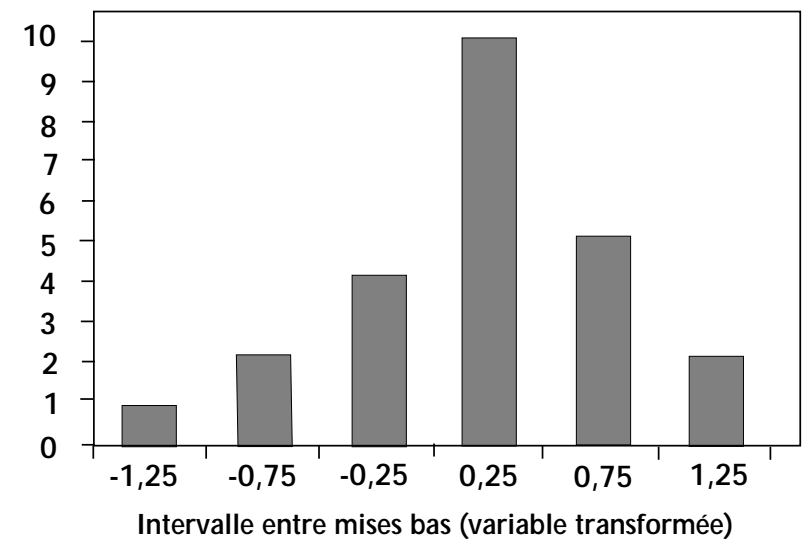

Effectifs

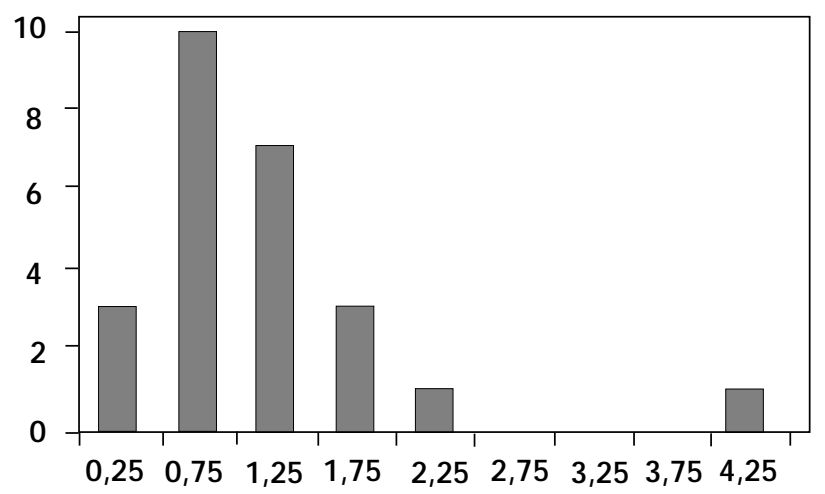

Age à la première mise bas (variable transformée)

Pour l'âge à la première mise bas, les performances ont été meilleures lorsque la femelle était née en saison sèche (à l'exception des caprins de Kaymor où elles étaient meilleures en saison des pluies). On peut relier cette observation à la saisonnalité des naissances, comme dans le cas de l'intervalle entre mises bas. Ainsi une femelle née en saison sèche peut mettre bas la première fois à la saison sèche suivante au moment où ont lieu la majorité des naissances. Une femelle née pendant l'hivernage est trop jeune pour mettre bas à la saison sèche qui suit et attendra la saison sèche suivante. 

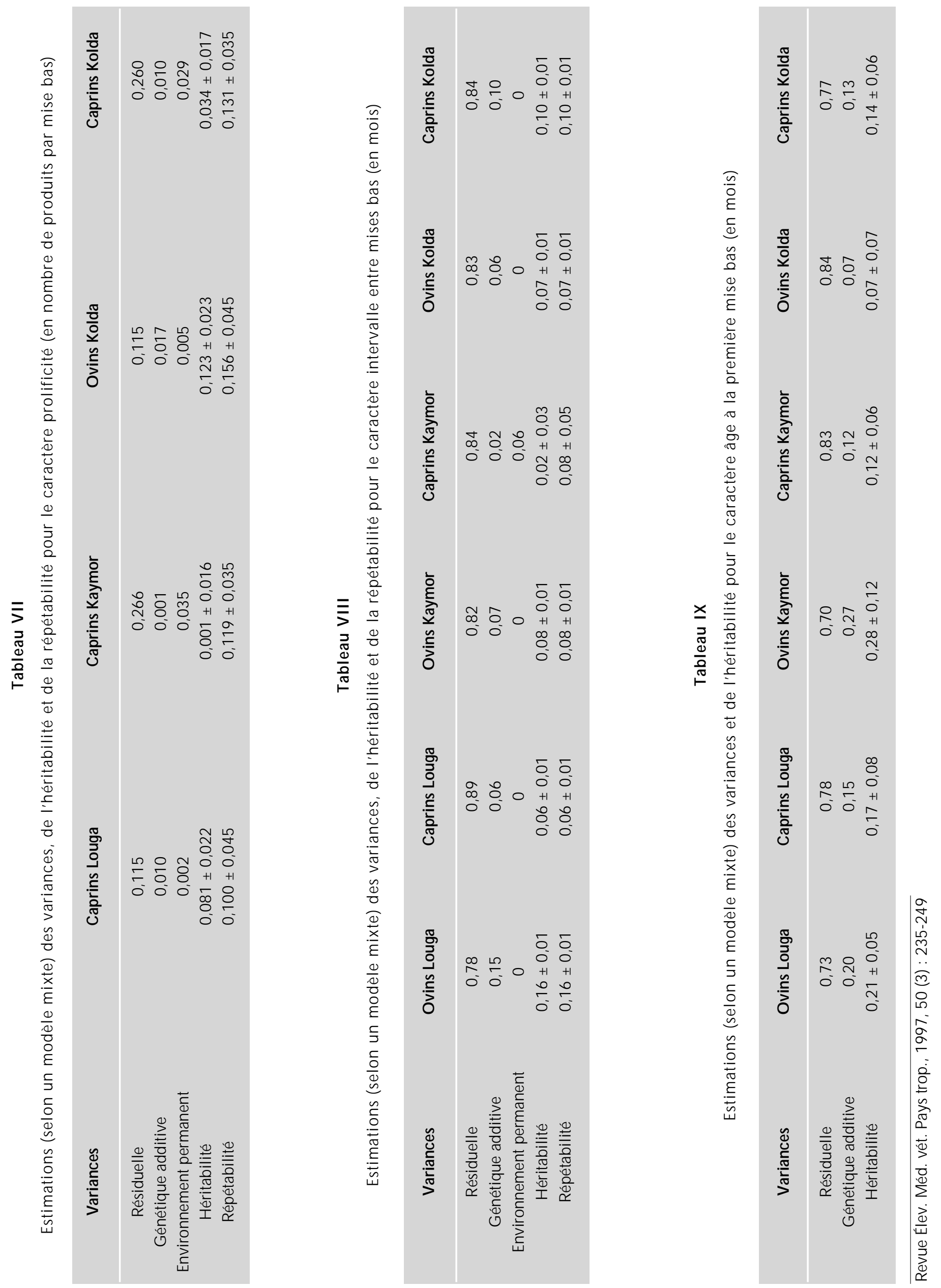


\section{Prolificité}

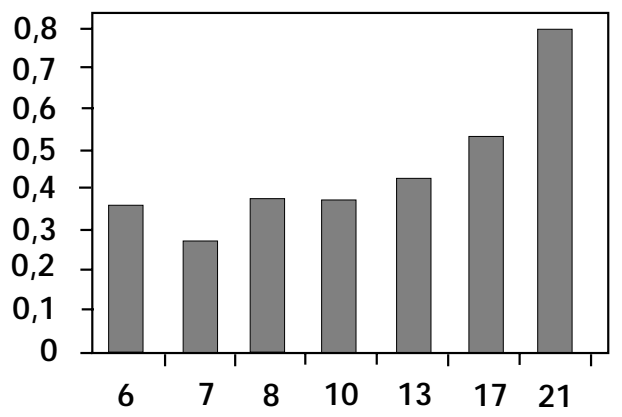

Age à la première mise bas (en mois)

\section{Prolificité}

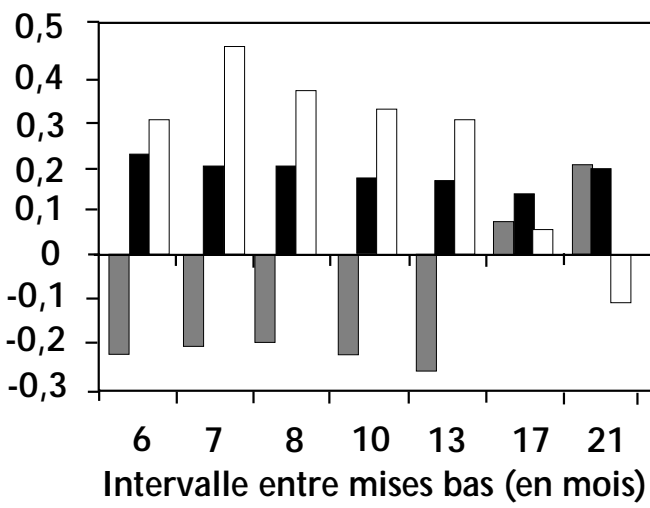

Figure 6 : histogramme des estimées de moindres carrés des modèles à effets fixes. Effets fixes âge à la première mise bas et intervalle entre mises bas, pour le caractère prolificité chez les caprins de la zone de suivi de Kaymor. (Blom G., 1958, NY, USA, John Wiley \& Sons).

L'importance des variations saisonnières sur les performances de reproduction des femelles est un phénomène général et on le retrouve dans de nombreuses études, notamment en milieu tropical $(14,27)$ où les animaux sont fortement dépendants des variations climatiques pour leur alimentation et leur rythme de reproduction.

\section{Effet de la précocité des femelles et de leur rang de mise bas} sur la prolificité

L'âge à la première mise bas a un effet significatif sur la prolificité : les femelles qui mettent bas tardivement ont des tailles de portée plus élevées et l'intervalle entre mises bas diminue lorsque le rang de mise bas augmente. De nombreux résultats allant dans ce sens ont été rapportés : le taux d'ovulation et la prolificité augmentent avec l'âge des femelles $(13,34)$. Cette observation es certainement liée à la croissance des femelles, puisque les premières naissances se produisent avant que celles-ci n'aient atteint leur poids adulte et il a été montré que la fertilité augmentait avec le poids des femelles $(17,33)$. A Kolda, par exemple, la première mise bas des caprins peut avoir lieu à un an, ce qui correspond à une fécondation à l'âge de 7 mois.

\section{Estimation de l'héritabilité et de la répétabilité}

Les valeurs estimées de l'héritabilité pour la prolificité ne dépassent pas 0,12. La répétabilité est de l'ordre de 0,16. L'héritabilité estimée sur la première mise bas est sensiblement supérieure à celle estimée sur la totalité des mises bas : elle varie entre 0,06 et 0,23.
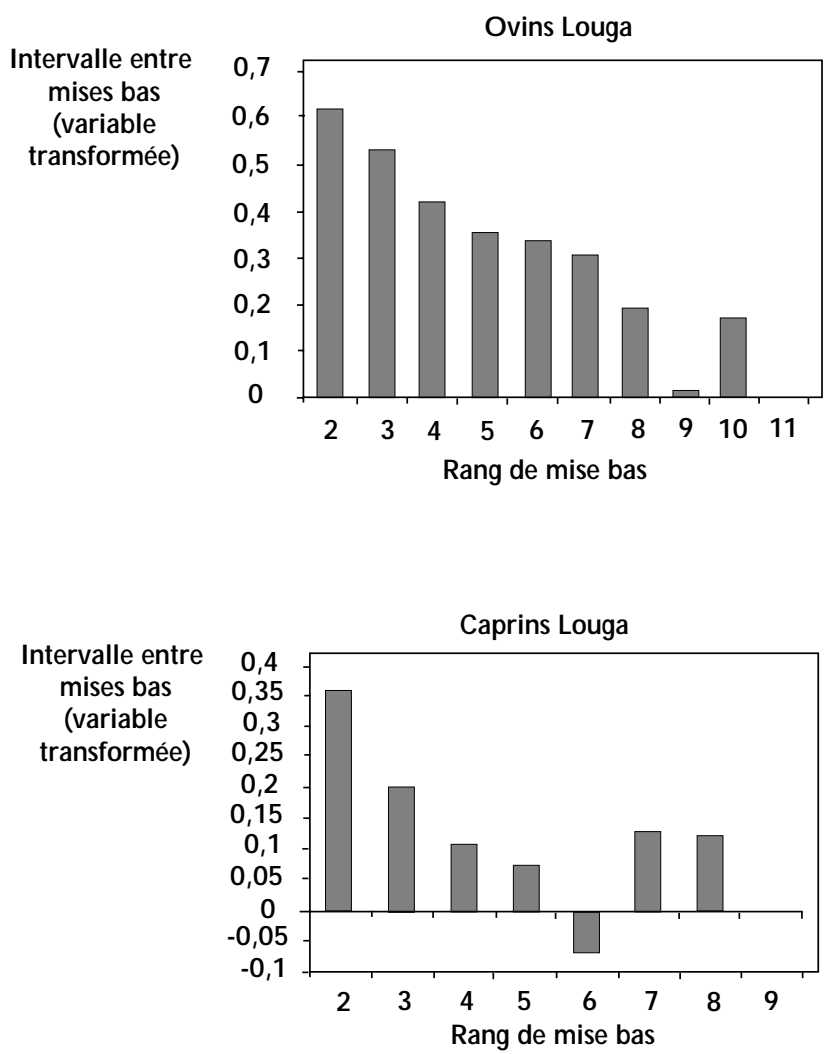

Figure 7 : histogramme des estimées de moindres carrés des modèles à effets fixes. Effet fixe rang de mise bas pour le caractère intervalle entre mises bas, chez les ovins et chez les caprins de la zone de suivi de Louga. (Blom G., 1958, NY, USA, John Wiley $\&$ Sons).

Les études faites sur d'autres races tropicales ont montré que la prolificité était un caractère peu héritable : l'héritabilité varie en général entre 0,04 et 0,26 chez les ovins (35). Les études conduites en milieu tempéré ont abouti à des héritabilités du même ordre de grandeur puisqu'elles se situent entre 0 et 0,25 quelle que soit la méthode d'estimation employée. Les héritabilités estimées sont de 0,03 chez des agnelles Lacaune (4), 0,07 chez la race Mérinos (24), entre 0,10 et $0,14 \mathrm{chez}$ la race Romney (1), 0,02 chez les agnelles et 0,23 chez les adultes Romanov (30). Enfin, dans la race Rambouillet, l'héritabilité varie entre 0,10 et 0,25 selon les auteurs $(16,31,34)$. Chez les caprins, encore assez peu d'études ont été faites à ce sujet. Mourad a trouvé une héritabilité de 0,03 chez une race ruandaise (20) et Odubote une héritabilité de 0,32 chez la race Naine d'Afrique de l'Ouest (West African Dwarf) (21) pour la prolificité. Ricordeau (28) donne une estimation moyenne de 0,08 pour l'héritabilité de la taille de portée des caprins chez diverses races vivant sous un climat tempéré.

En ce qui concerne la variabilité phénotypique dans les races sénégalaises, elle s'avère être du même ordre de grandeur que celle des races tempérées de même prolificité. Chez la race Mérinos d'Arles (5), de prolificité semblable à celle des Djallonké (soit 1,17 pour les Djallonké et 1,21 pour les Mérinos), le coefficient de variation est de 0,342, alors qu'il est de 0,330 chez les Djallonké. Chez les ovins Ile de France, de prolificité moyenne égale à 1,56, le coefficient est équivalent à 0,370 (5). Il est de 0,410 chez les chèvres Guinéennes, qui présentent la même prolificité. 
Les estimations trouvées pour l'héritabilité et la répétabilité de l'intervalle entre mises bas sont faibles, mais plusieurs facteurs de variation de ce caractère n'ont pas pu être pris en compte dans les analyses. Landais et coll. ont montré chez une race tropicale de bovins (race Baoulé), que l'intervalle entre mises bas dépendait de la survie du descendant issu de la mise bas précédente : le produit exercerait une influence négative sur la fertilité de la femelle en entretenant la lactation chez cette dernière (anœstrus de lactation) (14). Or, dans le cas du Sénégal, il n'a pas été tenu compte de la présence ou de l'absence du produit auprès de sa mère. La période de lutte n'étant pas fixe, il était difficile de savoir si les intervalles entre mises bas longs étaient provoqués par la présence du produit dans le troupeau ou s'ils étaient dus à d'autres causes (absence de mâles dans le troupeau, saisonnalité des naissances, etc.). Une autre source d'imprécision concerne les avortements : s'il y a avortement entre les deux mises bas considérées, l'intervalle entre mises bas est alors plus grand. Les avortements étant rarement déclarés, ils n'ont pas été pris en compte dans l'analyse de l'intervalle entre mises bas.

Les caractères de reproduction sont fortement soumis à la sélection puisqu'ils sont directement liés à la capacité d'adaptation des animaux. Il est possible aussi que la transmission génétique ne soit pas d'origine purement additive, mais fasse aussi intervenir des phénomènes de dominance, d'autant plus lorsqu'il s'agit de populations constituées de différentes races et croisements, comme à Kaymor.

Les résultats trouvés dans cette étude sont légèrement plus faibles que ceux obtenus dans d'autres travaux $(1,16,21,30,31,34)$. Cette différence peut avoir plusieurs origines :

- les performances ont été ajustées pour les facteurs fixes significatifs les plus facilement identifiables. Cependant, compte tenu de l'importance de la variabilité environnementale, des facteurs de milieu non contrôlés (par exemple un facteur troupeau intra-village ou des problèmes de pathologie affectant certains animaux non pris en compte avec la méthode utilisée) sont vraisemblablement à l'origine de variations importantes des performances de reproduction. Ces facteurs, s'ils ont été omis des analyses, peuvent être à l'origine d'un biais dans la valeur de l'héritabilité en surestimant la variance résiduelle ;

- l'importance de la consanguinité est difficile à estimer en l'absence de connaissance sur les paternités. L'effectif génétique des mâles est faible puisque la majorité est vendue avant l'âge de deux ans, et les mâles sont généralement originaires du troupeau dans lequel ils se trouvent. Cela ne permet pas d'exclure la possibilité de croisements consanguins qui pourraient être une cause de diminution de l'héritabilité. Cependant, la conduite sur parcours en troupeaux villageois, ainsi que la divagation des animaux autour des villages en saison sèche permettent un brassage génétique, ce qui laisse penser que la consanguinité doit être relativement faible. En l'absence d'une estimation plus précise de la consanguinité, les conséquences de celle-ci sur l'héritabilité doivent être envisagées ;

- dans un milieu trop contraignant, les femelles présentant les meilleures capacités reproductrices ne sont pas toujours capables de les exprimer de façon optimale. Il y aurait alors une corrélation négative entre la capacité à produire plus de descendants et la faculté à s'adapter aux contraintes de l'environnement, comme l'ont développé Bibé et coll. (2). Il s'agit dans ce cas d'une interaction génotype-milieu qui n'est pas prise en compte dans le modèle. Une autre explication serait que la sélection naturelle (voire la sélection par l'éleveur) ait contribué à éliminer les femelles les plus prolifiques si l'on suppose que ces dernières sont moins bien adaptées à des conditions de milieu difficiles. La capacité à produire un plus grand nombre de descendants dépendra des aptitudes d'élevage de la femelle : production laitière, qualités maternelles ;
- l'estimation de l'héritabilité en utilisant le seul lien de parenté mère-descendant ne permet pas de dissocier les effets génétiques directs des effets environnementaux que la mère exerce sur ses descendants (effets maternels). Selon plusieurs auteurs (7, 26, 29), la taille de la portée dont est issue la mère aurait un effet sur ses propres performances de reproduction : les brebis issues de portées simples auraient une prolificité supérieure à celles issues de portées multiples. Ainsi, une estimation reposant sur la seule parenté mère-descendant tendrait à sous-estimer les héritabilités, et ce d'autant plus que le milieu d'élevage présente de fortes contraintes.

\section{- CONCLUSION}

Le premier intérêt de cette étude réside dans l'analyse génétique de données recueillies dans des situations d'élevage villageois en zone tropicale. Malgré des généalogies incomplètes, le modèle d'analyse utilisé (modèle animal) a permis d'obtenir des estimations d'héritabilité cohérentes en comparaison avec la bibliographie, pour un premier groupe de variables liées à la productivité numérique des femelles.

Un autre intérêt de ce suivi en milieu traditionnel est le fait qu'il prend en compte les conditions réelles d'exploitation des animaux. Un contrôle de performance en station présente le risque de ne pas considérer l'interaction génotype-milieu qui intervient lorsqu'un individu présente des performances différentes selon le milieu dans lequel il se trouve. Cela peut se produire si le caractère étudié est lié à une capacité à utiliser le milieu ou à s'adapter à une composante défavorable de ce dernier. Ainsi le progrès génétique attendu dans le cas d'un programme de sélection peut être surestimé si le milieu dans lequel se fait le contrôle de performance est différent du milieu d'exploitation des animaux. Il est donc important, comme l'ont développé plusieurs auteurs $(2,6,25)$, de tenir compte à la fois des caractères de reproduction et des conditions d'adaptation des animaux, et d'orienter les recherches vers l'utilisation de races locales bien adaptées à leur environnement. Cela suppose d'étudier au préalable la pertinence des paramètres génétiques estimés dans ces conditions.

Sur la base des résultats de cette étude, et sous réserve que l'analyse des objectifs de sélection à conduire dans ce type d'élevage accorde la même importance à la productivité numérique que celle qui lui est donnée en milieu tempéré, une amélioration génétique serait envisageable. En effet, même avec des héritabilités plutôt faibles, il existe des exemples réussis de sélection pour l'augmentation de la taille de portée, les progrès étant d'autant plus élevés que la sélection a concerné à la fois les voies mâles et femelles. Ainsi, pour la race Lacaune, qui est sélectionnée pour la prolificité et la production laitière depuis plus de vingt ans, le progrès génétique réalisé sur la taille de portée est de 0,016 agneau par mise bas et par an (23).

Toutefois, il conviendrait de préciser qu'un schéma d'amélioration génétique dans de telles conditions de milieu ne peut être envisagé que s'il est accompagné d'une meilleure maîtrise du milieu, c'està-dire une amélioration des conditions sanitaires et alimentaires, et une gestion de la reproduction $(6,15,25)$. Dans le cas d'une amélioration de la prolificité, il est indispensable d'agir également sur les qualités maternelles et la production laitière qui vont de pair avec une augmentation de la productivité numérique. La prochaine étape d'analyse des données sera d'ailleurs l'estimation des paramètres génétiques de ce type de variable.

Aussi, un schéma d'amélioration génétique ne peut être considéré que dans le cadre d'un processus global d'amélioration des conditions d'élevage et d'une discussion sur les objectifs poursuivis. 


\section{BIBLIO GRAPH IE}

1. BAKER R.L., CLARKE J.N., CARTER A.H., DIPROSE G.D., 1982 Genetic and phenotypic relationships of hogget body weight and oestrus activity with reproduction in New Zealand Romney sheep. Proc. second World Congr. Genet. appl. Livest. Prod., 7: 519-524.

2. BIBE B., VISSAC B., 1979. Amélioration génétique et utilisation du territoire. In : Utilisation par les ruminants des pâturages d'altitude et parcours méditerranéens. Paris, France, IN RA, p. 480-491.

3. BLOM G., 1958. Statistical estimates and transformed beta variables, New-York, USA, John Wiley \& Sons.

4. BODIN L., 1979. Estimation des paramètres génétiques de la taille de portée des agnelles Lacaune après fécondation sur œstrus naturel et induit. Ann. Genet. Sel. Anim., 11 : 413-424.

5. BO DIN L., ELSEN J.M., 1989. Variability of litter size of French sheep breeds following natural or induced ovulation. Anim. Prod., 48 535-541.

6. BOUIX J., 1992. Adaptation des ovins aux conditions de milieu difficiles. Paris, France, INRA Productions animales, p. 179-184. (Hors série : Eléments de génétique quantitative et application aux populations animales)

7. DEWI I.A., OWEN J.B., EL-SHEIKH A., AXFORD R.F.E., BEIGINASSIRI M., 1996. Variation in ovulation rate and litter size of Cambridge sheep. Anim. Sci., 62: 489-495.

8. FAU GERE 0., D OCKES A.C., PERROT C., FAU GERE B., 1990 L'élevage traditionnel des petits ruminants au Sénégal. II. Pratiques de conduite et d'exploitation des animaux chez les éleveurs de la région de Louga. Revue Elev. Méd. vét. Pays trop., 43 : 261-273.

9. FAU GERE 0., D OCKES A.C., PERRO T C., FAU GERE B., 1990 L'élevage traditionnel des petits ruminants au Sénégal. I. Pratiques de conduite et d'exploitation des animaux chez les éleveurs de la région de Kolda. Revue Elev. M éd. vét. Pays trop., 43 : 249-259.

10. FAU GERE O., FAU GERE B., 1986. Suivi de troupeaux et contrôle de performances individuelles des petits ruminants en milieu traditionne africain. Aspects méthodologiques. Revue Elev. Méd. vét. Pays trop., 39 : 29-40.

11. FAU GERE O., FAU GERE B., 1993. Panurge: suivi individuel dans les systèmes d'élevage traditionnel, $2^{\mathrm{e}}$ ed. Maisons-Alfort, France, CIRAD-EM VT, $339 p$.

12. GROENEVELD E., 1996. REM L VCE. U ser's guide, vers. 3.2. Neustadt, Germany, Institute of Animal Husbandry and Animal Ethology, Federal Research Center of Agriculture, $52 \mathrm{p}$.

13. LAJOUS D., 1987. Mesure du taux d'ovulation et de la mortalité embryonnaire chez la brebis Romanov. U tilisation et intérêt de la cœlioscopie. Thèse doct., Institut national polytechnique, Toulouse, France, $71 p$.

14. LANDAIS E., POIVEY J.P., SEITZ J.L., 1980. Recherches sur la reproduction du cheptel taurin sédentaire du Nord de la Côte-d'Ivoire : utilisation des intervalles entre vêlages ; aspects méthodologiques et premiers résultats. Revue Elev. Méd. vét. Pays trop., 33 : 193-204.

15. LHOSTE P., 1980. L'amélioration génétique des zébus de I'Adamaoua (Cameroun) pour la production de la viande. In : Colloques sur l'élevage bovin en zone tropicale humide, Bouaké, Côte d'Ivoire, 18-22 avril 1977. Maisons-Alfort, France, IEMVT, p. 745-747.

16. MATOS C.A.P., THOMAS D.L., GIANO LA D., TEMPLEMAN R.J., YOUNG L.D., 1997. Genetic analysis of discrete reproductive traits in sheep using linear and non linear models. 1. Estimation of genetic parameters. J. Anim. Sci., 75: 76-87.

17. MBAYAHAGA J., BAUDOUX C., MANDIKI S.N.M., BISTER J.L., BRANCKAERT R., PAQUAY R., 1997. Paramètres de reproduction et de production des petits ruminants au Burundi. Anim. Genet. Res. Inf., 20 55-69.
18. MOULIN C.H., 1993. Performances animales et pratiques d'élevage en Afrique Sahélienne. La diversité du fonctionnement des troupeaux de petits ruminants dans la communauté rurale de $\mathrm{N}$ diagne (Sénégal). Thèse doct., IN RA, Paris-Grignon, France, $259 \mathrm{p}$.

19. MOULIN C.H., FAU GERE O., FAU GERE B., 1994. L'élevage traditionnel des petits ruminants au Sénégal. III Pratique de conduite et d'exploitation des animaux chez les éleveurs de la communauté rurale de Kaymor (Siné-Saloum, Sénégal). Revue Elev. Méd. vét. Pays trop., 47 : 223-234.

20. MOURAD M., 1994. Estimation of genetic and phenotypic parameters of some reproductive traits of African common goats in Rwanda. Small Rumin. Res., 15: 67-71.

21. ODU BO TE I.K., 1996. Genetic parameters for litter size at birth and kidding interval in the West African Dwarf goats. Small Rumin. Res., 20: 261-265.

22. PATTERSON H.D., THOMPSON R., 1971. Recovery of inter-block information when block-size are unequal. Biometrika, 58: 545-554.

23. PERRET G., BODIN L., MERCADIER M., 1992. Scheme for genetic improvement of reproductive abilities in Lacaune sheep. In: 43rd annual meeting of the European association for animal production, Madrid, Spain, september 14-17, 1992.

24. PIPER L.R., BINDON B.M., ATKINS K.D., ROGAN I.M., 1984. $O$ vulation rate as a selection criterion for improving litter size in Merino sheep. In: Lindsay D.R., Pearse D.J., eds. Reproduction in sheep. Canberra, Australia, Australian Academy of Science, Australian Wool Corporation, p. 237-239.

25. POIVEY J.P., 1987. Development of breeding methods in the tropics with limited availability of in-the-field recording systems. World Rev. Anim. Prod., 23: 83-92.

26. POIVEY J.P., COURNUT J., JULLIEN E., BIBE B., PERRET G., ELSEN J.M., BERNY F., BOUIX B., BODIN L., 1991. Estimation of meat sheep breeding values in French on farm performance recording system. In: 41st annual meeting of the EAAP, Toulouse, France, July 6-12, 1990

27. RAJAB M.H., CARTWRIGHT T.C., DAHM P.F., FIGUEIREDA E.A.P., 1992. Performance of three tropical hair sheep breeds. J. Anim. Sci., 70: 3351-3359.

28. RICORDEAU G., 1981. Genetics : breeding plans. In: Gall C., ed. Goat production. London, UK, Academic Press, p. 111-161.

29. RICORDEAU G., BODIN L., TCHAMITCHAN L., 1979. Amélioration de la prolificité des brebis par sélection. Revue bibliographique. In : $5^{\mathrm{e}}$ Journées de la recherche ovine et caprine, Paris, France, 5-6 décembre 1979. Paris, France, ITO VIC-SPEO C, p. 295-322.

30. RICORDEAU G., POIVEY J.P., LAJO US D., EYCHENNE F., 1986. Genetic aspects of ovulation rate and embryo mortality in Romanov ewes. Proc. third World Congr. Genet. appl. Livest. Prod., 11: 90-95.

31. SHELTON M., MENZIES J.W., 1970. Repeatability and heritability of components of reproductive efficiency in fine-wool sheep. J. Anim. Sci., 30: 1-5.

32. STATISTICAL ANALYSIS SYSTEM, 1990. SAS U ser's guide: statistics. SAS Institute Cary, NC, USA, 1028 p.

33. THIM O NIER J., 1975. Etude de la puberté et de la saison sexuelle chez les races prolifiques et leurs croisements avec des races françaises. In : 1ères Journées de la recherche ovine et caprine, Paris, France, 2-4 décembre 1995. Paris, France, IN RA-ITOVIC, p. 18-37.

34. WALDRON D.F., THOMAS D.L., 1992. Increased litter size in Rambouillet sheep: I. Estimation of genetic parameter. J. Anim. Sci., 70: 3333-3344.

35. WILSON L.T., MURAYI T.H., ROCHA A., 1989. Indigenous African small ruminant strains with potentially high reproductive performance. Small Rumin. Res., 2: 107-117.

Reçu le 15.4.97, accepté le 20.11.97 


\section{Summary}

Clément V., Poivey J.P., Faugère 0 ., Tillard E., Lancelot R., Gueye A., Richard D., Bibé B. Study on the variability of small ruminants' reproductive traits in the traditional husbandry system in Senegal

As part of the ongoing PPR (Pathology and productivity of small ruminants) program by CIRAD-EMVT (Montpellier, France) and ISRA (Dakar, Senegal) a genetic analysis was carried out based on data collected during individual monitoring in the Senegalese traditional husbandry system. The study concerned three different areas and two species, sheep and goats. The aim was to estimate genetic parameters of reproductive traits: prolificity, lambing interval and age at first lambing. As the individual monitoring was conducted in the traditional husbandry system, environmental variability was high and the animals were subjected to harsh environmental constraints. The first step was to identify and test environmental factors affecting the reproductive performance. The fixed factors - village where the animals came from, season and year of lambing (traits of prolificity and lambing interval) and season and year of birth (trait of age at first lambing) - were highly significant. With regard to prolificity, the parity and lambing interval fixed factors were significant, with a litter size increasing with parity and longer lambing intervals. The step of estimating heritability and repeatability was performed according to an "animal model" by estimating the variance components through restricted maximum likelihood. In sheep heritability was $0.12 \pm 0.02$ for prolificity; it varied from $0.07 \pm 0.02$ to $0.16 \pm 0.01$ for lambing interval and from $0.07 \pm 0.07$ to $0.28 \pm 0.12$ for age at first lambing. In goats, heritability varied from 0 to $0.08 \pm 0.02$ for prolificity, from $0.02 \pm 0.03$ to $0.10 \pm 0.01$ for lambing interval and from $0.12 \pm 0.06$ and $0.17 \pm 0.08$ for age at first lambing. The results were not as high as those in the references. This could be explained by the fact that the reproductive traits, even if they were corrected for the significant fixed factors, could be influenced by not controlled environmental factors. There may also be a conflict between a large offspring production and the ability to adapt to prevalent environmental conditions in Senegal.

Key words: Sheep - Goat - Reproductive performance Genetic parameter - Parturition interval - Age - Environmental factor - Season - Region - Senegal.

\section{Resumen}

Clément V., Poivey J.P., Faugère 0 ., Tillard $E_{\text {., }}$ Lancelot $\mathbf{R}$., Gueye A., Richard D., Bibé B. Estudio de la variabilidad de los caracteres de reproducción en los pequeños rumiantes en un medio de crianza tradicional en Senegal

Se realizó un análisis genético a partir de datos recolectados durante un seguimiento individual, establecido en un medio tradicional de Senegal, en el cuadro del programa de PPR (Patología y productividad de los pequeños rumiantes), por el CIRAD -EM VT (Centro de cooperación internacional en investigaciones agronómicas para el desarrollo, departamento de Crianza y de medicina veterinaria) y el ISRA (Instituto senegalés de investigaciones agrícolas). El estudio se llevó a cabo en tres regiones diferentes, en dos especies: ovinos y caprinos. El objetivo fue el de estimar los parámetros genéticos de los caracteres de reproducción de los animales: prolificidad, intervalo entre partos y edad al primer parto. Debido a que el seguimiento individual se realizó en un medio tradicional, la variabilidad ambiental fue importante y los animales estuvieron sometidos a condiciones ambientales difíciles. La primera etapa fue la de identificar y probar (gracias a modelos de análisi de varianza) los factores ambientales de variación de los rendimientos reproductivos. Los factores fijos fueron: pueblo de origen del animal, estación y año de parto (para los caracteres prolificidad e intervalo entre partos) y estación y año de nacimiento (para el caracter edad al primer parto); éstos fueron altamente significativos. Para el caracter prolificidad, los factores fijos de rango de parto e intervalo entre partos fueron significativos, con tamaños de camadas que aumentaron con el rango de parto y que fueron más elevadas para un intervalo entre partos largo. La etapa de estimación de la heredabilidad y de la repetibilidad se realizó según un "modelo animal", realizando la estimación de los componentes de la varianza por el máximo de similitud restringida. En los ovinos, la heredabilidad fue de $0,12 \pm 0,02$ para la prolificidad; variando entre $0,07 \pm 0,02$ y $0,16 \pm 0,01$ para el intervalo entre partos y entre $0,07 \pm 0,07$ y $0,28 \pm 0,12$ para la edad al primer parto. En los caprinos, la heredabilidad varió entre 0 y $0,08 \pm 0,02$, para la prolificidad, entre 0,02 $\pm 0,03 \mathrm{y}$ $0,10 \pm 0,01$ para el intervalo entre partos y entre $0,12 \pm 0,06$ y $0,17 \pm 0,08$ para la edad al primer parto. Los resultados obtenidos fueron menos elevados que los encontrados en la bibliografía. Esto podría explicarse por el hecho de que los rendimientos de reproducción, incluso corregidos para los factores fijos significativos, estarían influenciados por factores ambientales no controlados. También cabe la posibilidad de que exista un antagonismo entre la producción de un gran número de descendientes y la capacidad de adaptación a las condiciones del medio prevalecientes en Senegal.

Palabras clave: Ovino - Caprino - Reproductividad Parámetro genético - Intervalo entre partos - Edad - Factor ambiental - Estación del año - Región - Senegal. 